60-61| 2018

La culture dans l'enseignement du français langue étrangère: conceptions théoriques, programmes et manuels auX $\mathrm{XIX}^{\mathrm{e}}$ et $\mathrm{XX}^{\mathrm{e}}$ siècles

\title{
La formation culturelle des professeurs de français en Grèce (1914-1976) : enjeux et compromis d'une collaboration culturelle franco-hellénique
}

The cultural training of French teachers in Greece (1914-1976): issues and compromises of a Franco-Hellenic cultural collaboration

\section{Loukia Efthymiou}

\section{(2) OpenEdition \\ 1 Journals}

Édition électronique

URL : https://journals.openedition.org/dhfles/5456

DOI : $10.4000 /$ dhfles.5456

ISSN : 2221-4038

Éditeur

Société Internationale pour l'Histoire du Français Langue Étrangère ou Seconde

Édition imprimée

Date de publication : 1 janvier 2018

Pagination : 229-244

ISSN : 0992-7654

\section{Référence électronique}

Loukia Efthymiou, «La formation culturelle des professeurs de français en Grèce (1914-1976) : enjeux et compromis d'une collaboration culturelle franco-hellénique », Documents pour l'histoire du français langue étrangère ou seconde [En ligne], 60-61 | 2018, mis en ligne le 01 juin 2019, consulté le 28 mars 2023. URL : http://journals.openedition.org/dhfles/5456 ; DOI : https://doi.org/10.4000/dhfles.5456

Ce document a été généré automatiquement le 28 mars 2023.

Tous droits réservés 


\section{La formation culturelle des professeurs de français en Grèce (1914-1976) : enjeux et compromis d'une collaboration culturelle franco-hellénique}

The cultural training of French teachers in Greece (1914-1976): issues and compromises of a Franco-Hellenic cultural collaboration

\section{Loukia Efthymiou}

1 L'évolution des programmes de formation culturelle des francisants grecs au $\mathrm{XX}^{\mathrm{e}}$ siècle, intimement liée aux divers projets, créations et réformes relatives à l'organisation d'une préparation initiale, présente un intérêt tout à fait particulier. En raison, tout d'abord, des voies originales qu'emprunta la constitution progressive du champ disciplinaire en question en Grèce ; de la forte dimension politique dont elle est investie également. Fruits d'une collaboration franco-hellénique étroite mais non moins ambiguë, ces programmes constituent, d'une certaine manière, le reflet des fluctuations des relations diplomatiques et culturelles entre les deux pays.

Relevant, donc, aussi bien de l'histoire de l'éducation que de l'histoire de la diplomatie culturelle, la présente étude se propose, dans un premier temps, de suivre le processus de disciplinarisation académique des lettres françaises en Grèce à travers l'examen du contenu des programmes des différentes structures de formation, ébauchées ou mises en place entre 1914 et 1976 ; de faire ressortir, dans un second temps, la philosophie qui les sous-tend; de repérer enfin les écarts entre l'élaboration et l'application du modèle disciplinaire qu'ils proposent, dans la mesure où ils sont révélateurs des enjeux impliqués, des heurts produits et des compromis effectués entre partenaires culturels souvent antagonistes. 


\section{Archéologie de la disciplinarisation du champ culturel pour francisants grecs (1914-1920)}

3 À l'origine des premières tentatives de disciplinarisation des savoirs culturels pour professeurs de français se trouve la décision du ministère de l'Instruction publique grec de solliciter, en 1897, la collaboration de l'École française d'Athènes en vue de combler un vide institutionnel gênant: l'absence de formation initiale destinée aux maîtres chargés, depuis 1836 pourtant, à la fois de l'enseignement de la langue française et de l'histoire générale dans les établissements secondaires helléniques (Antoniou 2012: 69-75; Efthymiou 2015a: 31-57). Le nouveau champ disciplinaire ne commença toutefois à se constituer qu'à partir de la seconde décennie du $\mathrm{XX}^{\mathrm{e}}$ siècle, parallèlement à la concrétisation des premières formules de préparation au professorat de français. Même si ces projets n'aboutirent pas à une création institutionnelle effective, ils contenaient en germe les fondements organisationnels sur lesquels allaient reposer les réalisations ultérieures.

Concrètement, en 1914, dans le cadre de la collaboration culturelle franco-hellénique favorisée par l'attitude francophile du gouvernement vénizéliste (Flitouris 2004 : 48), le ministre de l'Instruction publique Ioannis Tsirimokos entreprit de conclure un accord avantageux pour la Grèce : il présenta à ses partenaires culturels un projet de loi relatif à la fondation d'une école normale pour maitres francisants à la charge du gouvernement français, lequel se voyait cependant privé du monopole de formation des futurs enseignants grecs. Le texte posait, pour la première fois, le principe de partage : l'École française d'Athènes et la faculté des lettres athénienne étaient appelées à coopérer étroitement tout en respectant chacune les frontières bien délimitées de sa sphère d'attribution. En ce qui concerne les programmes d'études, en particulier, cette double responsabilité organisationnelle et spatiale - voulue, du moins partiellement, de niveau universitaire - se traduisait par la division du cursus en deux volets : grec, axé autour de matières culturelles et pédagogiques plus générales - telles que la "psychologie », la «morale », la "pédagogie », l'« histoire»-, et français, centré uniquement sur les enseignements de spécialisation, à savoir pour le domaine culturel, la « littérature » et la « civilisation » françaises, cette dernière bien démarquée déjà de l'enseignement historique ${ }^{1}$. La guerre interrompit brutalement le processus de disciplinarisation à peine amorcé.

5 Il se poursuivit, à partir de 1918, dans le cadre d'un modèle institutionnel différent cependant : celui d'une école normale placée entièrement sous le contrôle du ministère grec, qui finançait d'ailleurs le projet. La nouvelle structure fut instituée par la loi de 1920 qui limitait le rôle de la partie française au choix des professeurs chargés de la direction de l'école et de l'enseignement des matières de spécialité, dont la littérature, l'histoire de l'art et de la civilisation française (loi 2 246, du 30-06-1920). L'histoire faisait, là encore, partie des enseignements généraux non confiés à l'équipe française composée tout de même d'un professeur d'histoire-géographie et de deux professeurs de langue et de littérature. Concession importante: ces matières devaient être dispensées en français et «d'après les programmes en vigueur en France dans [les] Écoles Normales $»^{2}$. Ainsi, bien que l'école n'ouvrît jamais ses portes en raison de la conjoncture économique et politique défavorable, le principe de partage des savoirs, établi sur des critères d'ordre pédagogique et politique à la fois, était bel et bien 
consacré et voué à un grand avenir dans le domaine de la formation de francisants grecs polyvalents.

\section{Entre Cours spécial et faculté des lettres: enseignements culturels et conflits d'attributions (1930-1955)}

6 C'est la création de 1930 qui permit de passer des principes et de l'esquisse théorique d'une formation culturelle à sa mise en place. Résultat d'un compromis diplomatique et de politique éducative (Efthymiou 2015c), la loi 4 595, inspirée sans doute du projet d'avant-guerre de Tsirimokos, donna naissance à une structure située entre enseignement primaire supérieur et universitaire. Son organisation fut encore commandée par l'idée de division: spatiale, dans un premier temps (deux années à l'université athénienne, puis, deux années encore au Cours spécial de l'Institut d'études supérieures françaises), pédagogique ensuite (deux cycles d'études - en réalité, deux cursus bien compartimentés, axé chacun autour de matières générales ou spécialisées), linguistique et culturelle enfin ${ }^{3}$. Il en résulta une formation culturelle hybride, classique autant que moderne, grecque autant que française, visant à prévenir le monopole français dans le domaine et à apaiser les craintes de ceux qui s'opposaient à l'implication d'une institution étrangère dans la préparation professionnelle des maîtres de l'enseignement public ${ }^{4}$.

7 Pour ce qui est de son volet français, plus particulièrement, la loi de 1930 n'innovait pas. Les savoirs qui composaient le bagage culturel des professeurs semblent être cristallisés autour d'un ensemble de matières reposant sur la notion, en usage à l'époque, de " civilisation » : à savoir, l'histoire de l'art, la littérature et l'histoire de la civilisation française. Le champ culturel de la formation était ainsi définitivement constitué.

8 Cependant, la mise en œuvre de ce programme par les responsables du Cours spécial et notamment ses modifications ultérieures ne furent pas toujours conformes aux grandes lignes énoncées par la loi. Certes, la disciplinarisation de l'enseignement littéraire était confiée aux Français. Elle fut inspirée, selon le futur directeur de l'Institut français Octave Merlier, «de la tradition universitaire » française ${ }^{5}$ : le choix des œuvres et des questions d'histoire littéraire mises à l'étude valorisait surtout une littérature classique, véhicule privilégié de l'image d'une France source de la civilisation moderne occidentale $^{6}$. Une initiation aux exercices d'explication de texte et de composition, piliers de cet enseignement, était également prévue ${ }^{7}$.

9 Pourtant, des écarts par rapport à la convention franco-hellénique sont constatés au sujet des modules d'histoire de l'art et de civilisation française. Le premier, dont le contenu était axé sur une initiation générale à ce champ disciplinaire accompagnée d'un examen plus approfondi de l'art pictural français, fut introduit dans le cursus en substance après la guerre. Quant à l'histoire de la civilisation française, elle fut immédiatement transformée en un enseignement typiquement historique confié le plus souvent à des agrégés de cette spécialité. Jusqu'à la réforme du plan d'études du Cours spécial en 1948, un programme très vaste comprenait l'étude sans coupure de l'histoire politico-économique, sociale et culturelle de l'Hexagone "des origines » jusqu'« au lendemain de la Grande Guerre » ainsi que le traitement de la "géographie générale » 
et "régionale », humaine et économique (Efthymiou 2015a: 330-332). Puis, dans les années 1950, la matière fut considérablement révisée dans un souci à la fois d'approfondissement et d'allègement : l'initiation étant réservée au cycle préparant au Cours spécial, on se limitait désormais à l'étude de deux périodes historiques et de deux grandes régions françaises par année, dont le choix était fixé tous les deux ans ${ }^{8}$. Un dernier écart par rapport aux textes législatifs des années 1930 (loi nº 4595 de 1930, décret de 1934) fut l'introduction, à partir de 1938, de modules de culture antique, alors qu'à l'origine ces matières étaient explicitement réservées à l'université athénienne. Ils étaient destinés aux élèves du Cours spécial non issus de la faculté des lettres et n'ayant pas de ce fait droit au diplôme officiel de professeur de français. La direction de l'Institut espérait, ainsi, harmoniser leur bagage culturel avec celui des rares étudiants de la faculté inscrits au Cours et, par là, leur assurer, au moyen d'une éventuelle réforme législative, une nomination dans l'enseignement public.

Par l'importance attribuée aux enseignements de l'histoire et de la littérature grécoromaine, le programme d'études du Cours spécial s'écartait, tout d'abord, de la voie française de constitution disciplinaire du champ culturel: il se différenciait considérablement, par exemple, de celui de l'École de préparation des Professeurs de Français à l'étranger créée en 1920 au sein de la Sorbonne (Chevalier 2010 ; Coste 2010), qui avait pourtant servi, selon le témoignage de Merlier lui-même, de modèle à son élaboration'. Il violait, par surcroît, l'accord franco-hellénique qui reposait sur un partage bien délimité des attributions des parties contractantes.

11 Or la transgression de cette condition essentielle de la convention bilatérale devait être lourde de conséquences pour le devenir de la formation mise en place en 1930.

\section{L'ère de la culture gréco-française : apogée et dépassements (1955-1976)}

Cette violation fut en effet à l'origine d'une véritable polémique culturelle transnationale aux implications politiques importantes qui, dans le contexte de vive concurrence d'après-guerre, due également à l'offensive anglo-saxonne dans le domaine linguistique, finit par conduire à la création de structures de formation initiale placées sous la tutelle des facultés des lettres helléniques. La décision en question fit basculer des équilibres établis depuis plusieurs décennies. L'objectif principal était de prévenir des distorsions semblables à l'avenir.

La loi de 1954 ( $n^{\circ} 3$ 107, 29-12-54), fruit de longues et difficiles négociations entre les deux gouvernements, reflète ces préoccupations: la dichotomie pédagogique et spatiale ainsi que le partage institutionnel d'attributions furent explicitement abandonnés. À l'image des deux sections d'études anglaises instituées en 1951 aux universités d'Athènes et de Thessalonique fut créée, au sein de la faculté des lettres athénienne, une section française. Parallèlement, à Thessalonique (1954), en vertu de la loi de 1931 qui fondait un Institut de langues et littératures étrangères dépendant de la faculté des lettres, ouvrait ses portes une deuxième structure de formation (Efthymiou 2015b : 490-492). Désormais exclusivement universitaire, l'organisation de la préparation professionnelle des francisants passait entre des mains grecques. L'élaboration des programmes également. La partie française, sans droit d'accès aux 
instances de décision universitaires helléniques, se limitait à l'administration des sections et à l'enseignement du volet français de la formation.

Les programmes d'études publiés en 1953 (université de Thessalonique) et en 1955 (université d'Athènes) faisaient écho aux principes organisateurs de la formation: les deux cycles séparés de la période précédente avaient fusionné en un seul cursus certes, toujours divisé toutefois en deux volets composés, d'un côté, de matières de "culture générale " enseignées en grec (explication d'auteurs classiques et modernes grecs, d'auteurs de l'Antiquité latine, histoire, philosophie...) et, de l'autre, d'enseignements de spécialité (langue, littérature et civilisation françaises). Vu les contraintes d'espace, notre analyse se limitera ici aux seuls programmes de la section athénienne.

Avant 1976, ils connurent deux grandes révisions, la première réalisée dans le cadre du mouvement de réforme engagé depuis 1964 en Grèce (projets de 1964 et 196710) et la seconde sous la dictature des colonels et plus précisément au début des années 1970 (programmes de 1971 et 1974). Les modifications qu'elles apportèrent, d'une part, renforcèrent le poids des matières de spécialisation au détriment des enseignements généraux, puisque de 48 heures pour toute la durée du cursus on passa à 54, puis, à 57 heures contre 49,39 et 36 heures respectivement pour l'enseignement en grec; d'autre part, elles valorisèrent les modules de formation culturelle française face aux matières linguistiques qui conservaient toutefois leur prééminence $(18,20,24$ heures contre 30, 34, 33 heures).

$16 \mathrm{Au}$ niveau qualitatif, les évolutions quantitatives déjà mentionnées ne furent pas accompagnées d'inflexions majeures. Tout au long de la période étudiée, les savoirs culturels français notamment restèrent axés plutôt autour de l'enseignement de la « littérature » et de la « civilisation ». L'étude de leur contenu n'en présente pas moins un intérêt particulier dans la mesure où elle laisse percevoir les désaccords, les divergences de vue et les concessions mutuelles des deux partenaires impliquées dans une collaboration éducative fragile.

17 C'est l'enseignement historique, vecteur potentiel de propagande idéologique et culturelle, qui se trouva, tout de suite, au centre de la controverse diplomatique et pédagogique. Les universitaires grecs entendaient, cette fois-ci, faire respecter les clauses de la nouvelle convention, dont le programme de 1955 était le reflet: celui-ci leur réservait les modules d'histoire médiévale européenne, d'histoire de l'Occident, d'histoire de France et de la Grèce moderne, alors qu'à l'image de ce que prévoyaient les accords bilatéraux précédents, il confinait les Français à l'organisation du champ disciplinaire, vague et imprécis et, de ce fait, plutôt anodin, de civilisation française. Les responsables de la diplomatie culturelle de l'Hexagone durent se résigner à accepter cet " arrangement » que le directeur de l'Institut Merlier se plaisait à appeler dans sa correspondance administrative le « sabotage de la civilisation française »"

18 À partir de 1955, une série de littéraires, professeurs d'université et lecteurs français, s'employèrent à circonscrire le contenu des modules qui composeraient le « champ » de la civilisation française : cantonné aux dernières années ou bien étalé sur toute la durée du cursus, il obéissait à une logique soit d'initiation, comme par exemple le «Cours de culture générale » dispensé entre 1964 et 1967 par le professeur à la chaire de civilisation française et spécialiste de Nerval, Jean Richer, soit d'approfondissement, tels les modules consacrés à l'étude de périodes précises (Moyen Âge et XVI ${ }^{\mathrm{e}}$ siècle, $\mathrm{XVII}^{\mathrm{e}}$, XVIII ${ }^{\mathrm{e}}$ et XIX ${ }^{\mathrm{e}}$ siècles). Ils furent confiés aux lecteurs français et, plus tard, aux auxiliaires d'enseignement grecs. Les documents conservés aux archives du ministère 
des Affaires étrangères français et de la faculté des lettres athénienne montrent pourtant que l'enseignement fut souvent situé à mi-chemin entre littérature et histoire de l'art, discipline, d'ailleurs, non incluse explicitement dans le programme :

J'avais chargé Monsieur Merlant, écrivait le directeur français de la Section Francis Pruner en 1958, d'initier les étudiants de première année à la civilisation française du Moyen Âge non par un cours magistral, mais par une présentation vivante d'images, de photographies, de projections lumineuses ou de films consacrés aux cathédrales romanes ou gothiques. C'était, il me semble, une façon de présenter la France [...] d'aujourd'hui [...]. (ADC, DGRCST, Enseignement, 1948-1961, 252 : Rapport de fin d'année de F. Pruner, 05-07-1958)

Des exceptions sont pourtant repérables : par exemple, en 1957, le directeur Descotes donna provisoirement une orientation historique au module «Introduction aux études françaises » abordé, jusque-là, à travers un prisme littéraire. Son objectif fut de parler d'abord du passé de la France [...]. J'initierai ensuite, ajoutait-il, ces étudiants au fonctionnement du système constitutionnel (est-il admissible, par exemple, qu'un étudiant ignore [...] pourquoi la République est la Quatrième ?), des grands organismes de l'État etc. Il va sans dire que le temps manquera pour approfondir ces questions. (Ibid. : Rapport de M. Descotes à l'ambassadeur de France, 20-12-1957)

C'est que cet universitaire était convaincu que «la littérature n'est pas un domaine isolé, sans rapports, par exemple, avec l'histoire ». En effet, il semble que d'une manière générale le lien entre texte et contexte était plutôt distendu dans l'organisation de l'enseignement littéraire du cursus, d'autant que souvent leur coordination chronologique y faisait défaut ${ }^{12}$. Citons, à titre d'exemple l'emploi du temps de l'année universitaire 1975-1976 ${ }^{13}$. Quatre modules de civilisation y étaient proposés ; leur choix révèle un certain flou théorique et méthodologique dans l'approche de ce champ disciplinaire. Chronologiquement, ils couvraient la période " contemporaine », les XVI ${ }^{\mathrm{e}}$ et $\mathrm{XVIII}^{\mathrm{e}}$ siècles. Plus précisément, en deuxième année, on étudiait selon le vaste intitulé/fourre-tout l'«Image de la France contemporaine: art, société, industrie, économie » et en quatrième, l'" Histoire de l'art, XVI ${ }^{e}$ et XVIII ${ }^{\mathrm{e}}$ siècles et 1890-1940", " L'humanisme de Ronsard, Rabelais, Montaigne, La Pléiade, Du Bellay, d'Aubigné » et «La crise dans la littérature contemporaine (1890-1960): Claudel, Mauriac, Artaud, Montherlant, Ionesco, Beckett » - modules qui, dans leur grande majorité, auraient pu tout aussi bien être inclus dans le programme littéraire. Parallèlement, en littérature, étaient abordées de la première à la quatrième année des œuvres et des questions touchant aux XVI ${ }^{\mathrm{e}}\left(4^{\mathrm{e}}\right.$ année), XVII ${ }^{\mathrm{e}}\left(3^{\mathrm{e}}\right.$ année) XVIII ${ }^{\mathrm{e}}\left(4^{\mathrm{e}}\right.$ année), XIX ${ }^{\mathrm{e}}\left(1^{\mathrm{e}}\right.$ et $2^{\mathrm{e}}$ années) et $\mathrm{XX}^{\mathrm{e}}$ siècles. Il en ressort que l'enseignement de la «civilisation » ne fonctionnait pas toujours comme support à l'étude des grandes œuvres littéraires.

21 Le choix de celles-ci ne permet pas non plus de percevoir une logique bien définie dans la constitution du programme littéraire. Des contraintes d'ordre pratique (nécessité de réunir les étudiants et les étudiantes de deux années successives, par exemple) mais aussi le fréquent changement de direction française ${ }^{14}$ sont parmi les principaux facteurs qui pourraient expliquer une certaine incohérence dans les choix et les priorités organisationnels. Concrètement, la planification soit reposait - du moins partiellement - sur le principe de progression graduelle, afin « que l'étudiant, au bout de trois ans d'études, ait une connaissance générale du développement de (la) littérature ${ }^{15}$, soit privilégiait l'étude détaillée de textes et de questions précises sans souci d'ordre chronologique évident et de progression logique apparente d'une année académique à l'autre. 
Ainsi, sous la direction Guyard (1955-1957), on passait, en deuxième année, de l'étude de la production littéraire des XVII ${ }^{e}$ et XVIII ${ }^{e}$ siècles en 1955 , à celle du XVII ${ }^{e}$ siècle uniquement en 1956, pour aboutir à un panorama de la littérature française en 1957, comprenant tous les siècles depuis le XVI ${ }^{\mathrm{e}}$. Mêmes constatations pour l'organisation du cours en troisième année : étude du XIX ${ }^{e}$ siècle en 1956, des XVIII ${ }^{e}, \mathrm{XIX}^{\mathrm{e}}$ et $\mathrm{XX}^{\mathrm{e}}$ siècles en $1957^{16}$. En quatrième année, le cours prenait la forme d'explication d'œuvres représentatives de tous les siècles, sorte de florilège de grands classiques (Lamartine, les Essais de Montaigne, L'Annonce faite à Marie de Claudel, Chrétien de Troyes, les Rêveries de Rousseau), dont les critères scientifiques et pédagogiques du choix ne sont pas évidents. Ils répondaient pourtant aux impératifs culturels qui organisaient l'enseignement littéraire à l'étranger depuis le XIX ${ }^{e}$ siècle : promotion d'une littérature classique, vecteur privilégié de diffusion de la pensée et de la culture françaises. Contrairement à Guyard, le directeur Jean-Hervé Donnard (1963-1967) adopta dans les années 1960 une approche chronologique de la production littéraire allant du XVII au $\mathrm{XIX}^{\mathrm{e}}$ siècle : l'étude des œuvres représentatives les plus importantes fut désormais étalée sur les trois dernières années du cursus (Efthymiou 2015a : 280-281).

À l'issue de plusieurs enquêtes pédagogiques et d'un débat ouvert à partir de 1961, la réforme de 1971 apporta deux modifications majeures dans l'organisation pédagogique de la formation. Elle introduisit dans le programme grec le système des modules obligatoires à option, divisa le volet français du cursus en deux cycles, linguistique et culturel, et confina les modules de littérature et de civilisation aux deux dernières années de la formation. Du moins en théorie, car l'examen des sources révèle que la direction française n'a pas toujours respecté la structure bipolaire voulue par les concepteurs du nouveau programme : dans la pratique, « littérature » et « civilisation » étaient enseignées tout au long des quatre années d'études ${ }^{17}$. À notre connaissance, il n'y eut cependant aucune réaction de la part des universitaires grecs à cette nouvelle transgression française des politiques officielles, preuve que l'ère des conflits et des oppositions était désormais plutôt révolue.

\section{Esquisse d'une conclusion ou la cristallisation d'une identité disciplinaire francisante}

Le dernier directeur français quitta la Section en 1976, au lendemain du rétablissement de la République en Grèce. Son départ marque un temps fort de la collaboration francohellénique dans ce domaine qui durait déjà depuis près d'un siècle ${ }^{18}$.

Celle-ci avait été jusque-là étroite, mais, en même temps, conflictuelle et, par là même, fragile. Elle n'en avait pas moins contribué à mettre sur pied et à consolider une formation initiale pour francisants ; à constituer également le "champ » du français langue étrangère en Grèce. L'enseignement de la culture en faisait, tout naturellement, partie. Certes, depuis l'élaboration du premier projet en 1914 jusqu'à la publication des programmes des années 1970, sa disciplinarisation reposa sur le même principe : celui qui appelait à une structure bipolaire axée, pour des raisons d'ordre diplomatique, idéologique, culturel et professionnel, sur les lettres grecques et françaises à la fois. Mais elle connut également, dans son cheminement vers "l'universitarisation", des évolutions et des adaptations intéressantes liées toujours à des compromis politiques et à des impératifs pédagogiques. 
Ironie de l'histoire, au moment où commençait le départ des universitaires français de la Section, le programme de spécialisation dominait, pour la première fois, sur le volet grec. En outre, la réforme de 1976 proposait une planification de l'enseignement culturel français plus cohérente, puisque l'étude du contexte socio-politique et idéologique d'une période historique sous-tendait désormais celle de sa production littéraire. La littérarisation de ce champ disciplinaire se rétrécit au profit d'un enseignement de la "civilisation française " désormais historicisé qui gagnait, d'un point de vue quantitatif, en importance. Resté en vigueur pendant plus de trente ans, ce programme devait contribuer à jeter les bases du développement ultérieur des domaines de spécialisation à l'origine des sections de littérature et d'histoire de la civilisation françaises du DLLF athénien (Efthymiou 2015a : 293-294) ${ }^{19}$.

\section{BIBLIOGRAPHIE}

Sources primaires

Archives

Archives Diplomatiques, site de la Courneuve (ADC), Service des CEuvres Françaises à l'Étranger (SOFE), dossier 188 ; Direction générale des affaires culturelles, scientifiques et techniques (DGRCST), Enseignement, 1948-1961, dossier 252.

Archives Diplomatiques de Nantes (ADN), Athènes. série A, dossiers 274, 301 ; archives de l'Institut français d'Athènes (AIFA), dossiers 1045, 1061, 1072, 1090.

Archives de la Faculté des lettres de l'Université d'Athènes (AFL). Programmes d'études 1958-1968, 1965-1969, 1970-1972, 1973-1979. Programme des auteurs, 1965-66 et Horaires et tableau de services, $1967-1968$.

Archives Historiques de l'Université d'Athènes (AHUA); Comptes rendus (CR) des assemblées générales

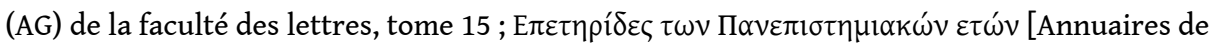
l'Université d'Athènes], 1955-1971.

Archives Nationales (AN) site Pierrefitte sur Seine : série F/17, 13598.

Sources imprimées

Loi 2246 du 24-06-1920, relative à la fondation d'une école normale destinée à la formation des professeurs de français, J.O., fasc. A, 144/30-06-1920 : 1 330-1 332.

Loi 4595 du 26-04-1930, relative la formation des professeurs de langue française, J.O., fasc. A, 138/02-05-1930: 1134 .

Loi 5139 du 10-07-1931, relative à la création d'un Institut de langues et littératures étrangères à l'Université de Thessalonique, J.O., fasc. A, 207/16-07-1931 : 1 485-1 486.

Décret présidentiel du 29-06-1934, relatif aux examens de fin d'études du Cours Spécial de l'Institut français, J.O., fasc. A, 223/17-07-1934: 1324. 
Décret royal du 24-11-1953, relatif aux modules obligatoires des étudiants de l'Institut de Langues et Littératures étrangères de l'Université de Thessalonique concernant la langue et la littérature françaises, J.O., fasc. A, 348/19-12-53 : 2 820-2 821.

Loi 3107 du 29-12-54, relative à la création de la Section d'Études françaises auprès de la Faculté des Lettres de l'Université d'Athènes, J.O., fasc. A, 314/30-12-54 : 2573.

Décret royal du 03-02-1955, relatif aux modules obligatoires et aux examens de la Section d'Études françaises de la Faculté des Lettres de l'Université d'Athènes, J.O., fasc. A, 25/04-02-55 : 131-132.

Arrêté ministériel 17 6512, du 21-12-71, relatif au programme de la Faculté des lettres de l'Université d'Athènes, J.O., fasc. B, 1 031/23-12-1971 : 7812.

Arrêté ministériel 67578 du 16-10-74, relatif aux modules enseignés et examinés à la Faculté des lettres de l'Université d'Athènes, J.O., fasc. B, 1 092/26-10-1974: 8 639-8 640.

Arrêté ministériel 8095 683/15 510 du 03-09-76, relatif aux modules enseignés et examinés à la Faculté des lettres de l'Université d'Athènes, J.O., fasc. B, 1 155/18-09-1976 : 9 196-9 196.

Sources secondaires

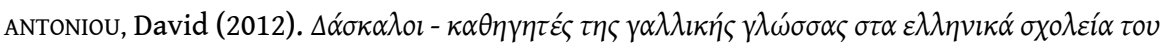

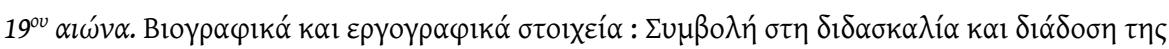

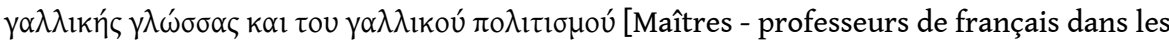
établissements grecs du XIX ${ }^{\mathrm{e}}$ siècle. Éléments biobibliographiques : Contribution à l'enseignement et à la diffusion de la langue et de la civilisation françaises]. Athènes : Centre international de recherche - La Fontaine.

CHEVALIER, Jean-Claude (2010). « Ferdinand Brunot et les débuts de l'École de préparation des professeurs de français à l'étranger ». Documents pour l'histoire du français langue étrangère ou seconde, 44, 15-27. En ligne : [https://journals.openedition.org/dhfles/2727].

COSTE, Daniel (2010). « Note sur la création de l'École de préparation des professeurs de français à l'étranger ». Documents pour l'histoire du français langue étrangère ou seconde, 44, 29-39. En ligne : [https://journals.openedition.org/dhfles/2739].

EFTHYMIOU, Loukia (2011). « Histoire d'une cohabitation, histoire d'une collaboration : les personnels grec et français du Département de Langue et Littérature françaises de l'Université d'Athènes (1954-1991) ». In Argyro Proscolli et al. Communiquer, échanger, collaborer en français dans l'espace méditerranéen et balkanique, Actes du $7^{\mathrm{e}}$ Congrès Panhellénique et International des Professeurs de Français. Athènes, Université d'Athènes, 215-226.

EFTHYMIOU, Loukia (2015a). La formation des francisants en Grèce : 1836-1982. Paris : Publibook. EFTHYMIOU, Loukia (2015b). « La chaire de littérature française à l'université de Thessalonique. Histoire d'une institution franco-hellénique culturelle, 1925-1954 ». In Frideriki Tabaki-Iona et al. (dir.) Médiation et réception dans l'espace culturel franco-hellénique, actes du colloque international organisé les 13, 14 et 15 décembre 2013. Athènes : Aigokeros, 486-499.

EFTHYMIOU, Loukia (2015c). « Le rôle de l'École française d'Athènes dans l'institution d'une formation de francisants en Grèce, 1897-1930 ». Documents pour l'histoire du français langue étrangère ou seconde, 55, 121-136. En ligne :

[https://journals.openedition.org/dhfles/4298]. 
FLITOURIS, Lampros (2004). À la recherche d'une véritable politique culturelle internationale. La présence culturelle et spirituelle de la France en Grèce de la fin de la Grande Guerre aux années 1960. Thèse de doctorat, Université de Versailles Saint-Quentin-en Yvelines (344 p.).

PROSCOLli, Argyro (2008). « Les efforts d'actualisation du Programme d'Études du Département de Langue et Littérature françaises de l'Université d'Athènes ». In Stefanos Vlachopoulos \& Themistocles Gogas (dir.). Foreign Language Teaching in Tertiary Education, Proceedings of the $2^{\text {nd }}$ International Conference. Ioannina : Carpe Diem, 27-41.

\section{NOTES}

1. ADN, Athènes, série A, 274 : note $n^{\circ} 22023,15-07-1914$, accompagnée d'un projet de loi du ministre de l'Instruction publique I. Tsirimokos. AN/F17/13598: Rapport du directeur sur les travaux de l'ÉFA, 1913-1914.

2. ADN, Athènes, série A, 301 : note du ministre des Affaires étrangères français, 30-01-1919.

3. «Art. I. Les étudiants de l'École de Philosophie [...] de nos Universités qui ont subi [les] examens [...] de la deuxième année universitaire, peuvent être admis au diplôme de langue française, s'ils ont suivi au moins pendant deux années les cours du cycle spécial et subi avec succès les examens sur les matières suivantes: Art. II. [...] la langue française et la littérature, l'histoire générale, l'histoire de la civilisation française et des arts en France et la Pédagogie. La langue française, la littérature et l'histoire de la civilisation française sont enseignées à une Section spéciale par les membres de l'École française d'Archéologie [...]; les autres matières sont enseignées par les professeurs de l'Université. » Loi 4 595, 26-04-1930. ADC, SOFE 188 : texte de loi traduit par les soins des autorités diplomatiques françaises.

4. AHUA, CR, t. 15, AG 7e/15-06-1930: 144-145.

5. ADN, Athènes, archives IFA, 1090 : Roger Milliex, « La préparation au professorat de français », Institut Français d'Athènes, Congrès de l'enseignement du français, Pâques 1950 (1-4 avril), p. 137.

6. P. ex. Les Précieuses ridicules de Molière, Les Rayons et les Ombres de Victor Hugo, Les Essais de Montaigne, Les Pensées, de Pascal ; Boileau et la doctrine classique, Montaigne et l'humanisme, Parnasse et symbolisme etc. (Efthymiou 2015a : 325-328).

7. Les principaux axes de cette planification se retrouvent également dans les programmes de littérature française élaborés par différents Instituts français. ADN, Athènes, archives IFA, 1045 : Programmes de littérature française des Instituts Français de Naples, de Florence et du Centre d'Études Supérieures de Rome, doc. $n^{\circ}$ 121, 20-10-49.

8. Ibid., 1068 : «La préparation au professorat à l'Institut français d'Athènes. Note établie par M. Milliex », 18-02-1956.

9. Ibid., 1072: «Rapport sur l'activité de l'Institut français d'Athènes pendant l'année 1959-1960», fasc. I, 53.

10. AFL, Programmes 1965-1969 : projet de décision ministérielle d'après la proposition de la Faculté des Lettres, AG 07-12-1964; AG 11e/16-01-1967 : projet de décision ministérielle.

11. Ibid., 1061, dossier Matton : lettre $n^{\circ} 12$ 438, 17-04-1954.

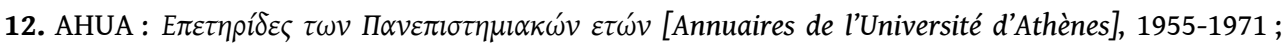
AFL, Programmes d'études 1958-1968 : 1960-1961 ; ibid., Programmes d'enseignement 1965-1969 : Programme des auteurs, 1965-1966 et Horaires et tableau de services, 1967-1968; ibid., Programmes d'enseignement 1970-1972 : 1970-1971. ADC, DGRCST, Enseignement, 1948-1961, 252: Rapport de fin d'année de F. Pruner, 05-07-1958 ; "Rentrée universitaire 1959-1960", rapport M. Descotes, 21-10-1959. Cf. Efthymiou 2015a : 276-282.

13. AFL, Programmes d'études 1973-1979 : 1975-1976. 
14. Six directeurs se succédèrent de 1955 à 1976 : Marius-François Guyard (1955-1957), Maurice Descotes (1957-1962), Jean-Hervé Donnard (1962-1967), Robert Jouanny (1967-1971), Henri Bouiller (1971-1974), Nicolas Wagner (1974-1976).

15. AFL, CA, 1959-1960, 501-700 : Rapport M. Descotes, 21-12-1959, doc. cité.

16. Leurs intitulés plutôt vagues $\left(\mathrm{XIX}^{\mathrm{e}}, \mathrm{XVIII}^{\mathrm{e}}, \mathrm{XVII}^{\mathrm{e}}\right.$... siècles) ne permettent pas de se faire une idée précise du contenu des cours.

17. AFL, Programmes d'études 1973-1979 : 1975-1976, doc. cité.

18. Des lecteurs français allaient continuer à offrir quelques heures d'enseignement jusqu'en 1991 (Efthymiou 2011).

19. Département de Langue et Littérature françaises. Pour une analyse critique des programmes du DLLF avant leur réforme en 2009, cf. Proscolli 2008.

\section{RÉSUMÉS}

Intimement liée aux divers projets, créations et réformes relatives à l'organisation d'une formation initiale, l'évolution des programmes de formation culturelle des francisants grecs au XXe siècle doit être étudiée dans une double optique. Celle, tout d'abord, que proposent les voies originales qu'emprunta le processus de disciplinarisation des études françaises en Grèce. Cette approche est, par ailleurs, inséparable d'une autre, plus politique : fruits d'une collaboration franco-hellénique étroite autant qu'ambiguë, les programmes d'enseignement des structures projetées ou mises en place entre 1914 et 1976 constituent, d'une certaine manière, le reflet des fluctuations des relations diplomatiques et culturelles entre les deux pays.

Intimately linked with the various projects, creations and reforms related to the organization of an initial training, the development of the cultural curricula of the Greek Teachers of French in the twentieth century must be studied in a dual perspective. Primarily the one that is proposed by the original ways that borrow the process of disciplinarization of French studies in Greece. This approach is, moreover, inseparable from another, more political: fruits of close as well as ambiguous French-Hellenic cooperation, the educational programs of the structures designed or created between 1914 and 1976 are, in some way, the reflection of the fluctuations of diplomatic and cultural relations between the two countries.

\section{INDEX}

Mots-clés : disciplinarisation, culture, littérature, histoire de la civilisation, Institut français d'Athènes, Cours spécial, Université d'Athènes, programmes d'études

Keywords : disciplinarization, culture, literature, history of civilization, French Institute of Athens, training course for teachers of French, university of Athens, curriculum

\section{AUTEUR}

\section{LOUKIA EFTHYMIOU}

Université nationale et capodistrienne d'Athènes - leythim@frl.uoa.gr 